\title{
Activation of MAPKs influences the expression of drug-metabolizing enzymes in primary human hepatocytes
}

\author{
Petr Bachleda ${ }^{1}$, Radim Vrzal ${ }^{2}$ and Zdeněk Dvořák ${ }^{2}$ \\ ${ }^{1} 2^{\text {nd }}$ Department of Surgery, University Hospital Olomouc, I. P. Pavlova 6, 775 20 Olomouc, Czech Republic \\ ${ }^{2}$ Department of Cell Biology and Genetics, Faculty of Science, Palacky University, Šlechtitelů 11, 78371 Olomouc, Czech Republic
}

\begin{abstract}
We examined the effects of model activators of mitogen-activated protein kinases (MAPKs) on basal and rifampicin-, phenobarbital- and dioxin-inducible expression of phase I and phase II biotransformation enzymes in primary human hepatocytes. Cells were treated for $24 \mathrm{~h}$ with sorbitol (SOR), anisomycin (ANI) and epidermal growth factor (EGF) in the presence or absence of inducers. The levels of CYP1A1, CYP1A2, CYP2B6, CYP3A4, UGT1A1, UGT2B17, SULT1A1, SULT2A1, SULT1B2, GSTA1, GSTA2 mRNAs were determined. SOR and EGF inhibited the expression of the tested genes, while ANI had no effect. We conclude that MAPKs play important role in the transcriptional regulation of drug-metabolizing enzymes.
\end{abstract}

Key words: Xenoreceptors - MAPKs - Human hepatocytes - Cytochrome P450

The expression of genes involved in xenobiotic metabolism is regulated in particular by several xenoreceptors, i.e. aryl hydrocarbon receptor (AhR), constitutive androstane receptor (CAR), and pregnane $\mathrm{X}$ receptor (PXR). In addition, there exists crosstalk between xenoreceptors and other nuclear receptors or transcription factors controlling signaling pathways that regulate the homeostasis of endogenous compounds (Pascussi et al. 2008; Pavek and Dvorak 2008). However, the exact molecular mechanisms underlying the action of xenoreceptors are not fully understood yet. There is increasing body of evidence that phosphorylation of xenoreceptors, their transcriptional co-activators, co-repressors and target genes, plays a pivotal role in their function (Henklova et al. 2008b; Oesch-Bartlomowicz and Oesch 2008). In this view, protein kinases are potentially the important regulators of drug metabolism.

In the current paper, we examined the effects of mitogenactivated protein kinases (MAPKs) activators on the basal and inducible expression of several enzymes of phase I and phase II of biotransformation. Anisomycin (ANI), sorbitol (SOR) and epidermal growth factor (EGF) were used to activate p38 by genotoxic shock (Kyriakis and Avruch 1996), c-Jun-N-

Correspondence to: Zdeněk Dvořák, Department of Cell Biology and Genetics, Faculty of Science, Palacky University, Šlechtitelů 11, 78371 Olomouc, Czech Republic

E-mail: moulin@email.cz terminal kinase by osmotic shock (Bogoyevitch et al. 1995), and extra cellular-activated protein kinase via EGF-receptor (Roberts and Der 2007), respectively. As the representatives of phase I enzymes cytochromes $\mathrm{P} 450$ that are regulated preferentially at transcriptional level were determined: CYP1A1, CYP1A2, CYP2B6, CYP2C9 and CYP3A4. Following phase II enzymes were analyzed: uridine diphosphate-glucuronyl transferases (UGT1A1, UGT2B17), sulfotransferases (SULT2A1, SULT1A1, SULT1B2) and glutathione-S-transferases (GSTA1, GSTA2). In addition, we examined the effects of MAPKs activators on the expression of the receptors involved in transcriptional regulation of drug-metabolizing enzymes, i.e. AhR, PXR, CAR and glucocorticoid receptor (GR). The study was performed in primary cultures of human hepatocytes, which is the most respected model for studying drug metabolism in man (Donato et al. 2008).

Human hepatocytes were isolated and the cultures prepared as described elsewhere (Vrzal et al. 2008). Experiments were performed in human hepytocytes cultures FT278 and FT280. RT-PCR measurements were performed in duplicates. Isolation of total RNA, synthesis of cDNA and RTPCR determination were performed as described previously (Vrzal et al. 2008). Relative expression of individual mRNAs was normalized per GAPDH (glyceraldehyde-3-phosphate dehydrogenase) as housekeeping gene.

Human hepatocytes were treated for $24 \mathrm{~h}$ with model MAPKs activators SOR $(0.4 \mathrm{~mol} / \mathrm{l})$, ANI $(5 \mu \mathrm{mol} / \mathrm{l})$, EGF 

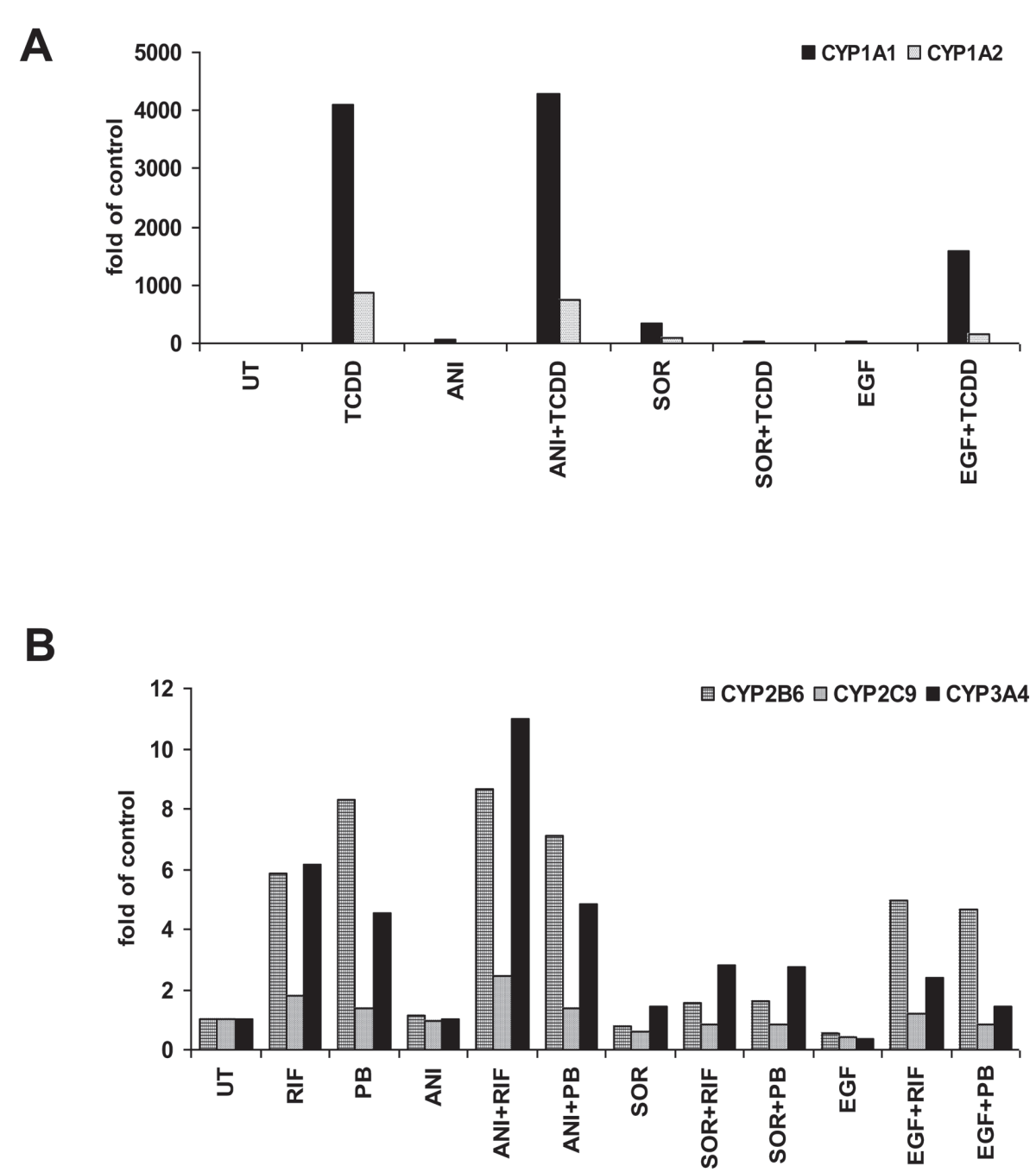

Figure 1. Effects of MAPKs activators on basal and inducible expression of CYP1A1, CYP1A2, CYP2B6, CYP2C9 and CYP3A4 mRNAs in human hepatocytes. Shown are RT-PCR analyses of CYP1A1, CYP1A2 (A), CYP2B6, CYP2C9 and CYP3A4 (B) mRNAs from human hepatocytes culture FT278. The data are mean from duplicate measurements and are expressed as fold induction over DMSO-treated cells. The data were normalized to GAPDH mRNA levels. UT, untreated cells.

$(75 \mathrm{ng} / \mathrm{ml})$ or vehicle (DMSO, $0.1 \% \mathrm{v} / \mathrm{v})$ in the presence or absence of model inducers rifampicin (RIF; $10 \mu \mathrm{mol} / \mathrm{l}$ ), phenobarbital (PB; $500 \mu \mathrm{mol} / \mathrm{l})$ or 2,3,7,8-tetrachlorodibenzop-dioxin (TCDD; $5 \mathrm{nmol} / \mathrm{l}$ ).

First we tested the effects of MAPKs activators on the basal and inducible expression of phase I enzymes. All MAPKs activators slightly elevated basal levels of CYP1A1 and CYP1A2 mRNAs. In contrast, SOR and EGF, but not ANI, drastically inhibited TCDD-mediated induction of CYP1A1 and CY1A2 mRNAs (Fig. 1A). SOR decreased basal expression of CYP2B6 and CY2C9 mRNAs. SOR also strongly inhibited both RIF- and PB-inducible expression of CYP2B6, CYP2C9 and CYP3A4 mRNAs. EGF decreased both basal and inducible expression of CYP2B6, CYP2C9 and CYP3A4 mRNAs. In contrast, ANI had no effect on basal and PB-inducible expression of CYP2B6, CYP2C9 and CYP3A4 mRNAs, while ANI augmented RIF-inducible expression of these genes (Fig. 1B).

Next we analyzed the effects of MAPKs activators on the expression of phase II enzymes. Basal levels of UGT1A1, UGT2B17, SULT2A1, SULT1A1, SULT1B2, GSTA1 and GSTA2 were diminished by SOR and EGF but not by ANI (Fig. 2). We observed induction of UGT1A1 but not UGT2B17 by RIF, PB and TCDD. This induction was inhibited by SOR, augmented by ANI, and unaffected by EGF (Fig. 2A). Expression of SULT1A1 and SULT1B2 was decreased by all tested inducers, while RIF and PB slightly induced SULT2A1. This induction was blocked by SOR and 
A

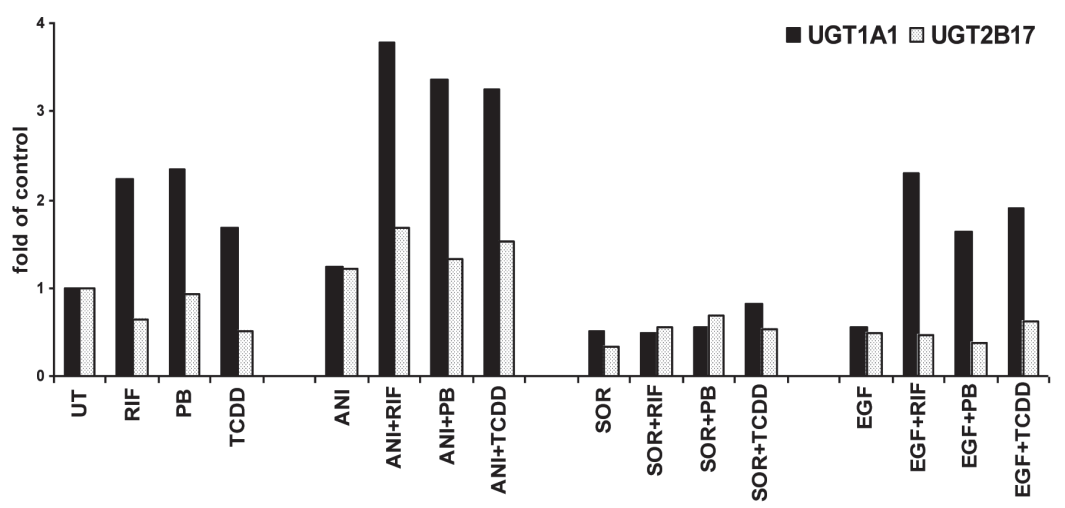

B
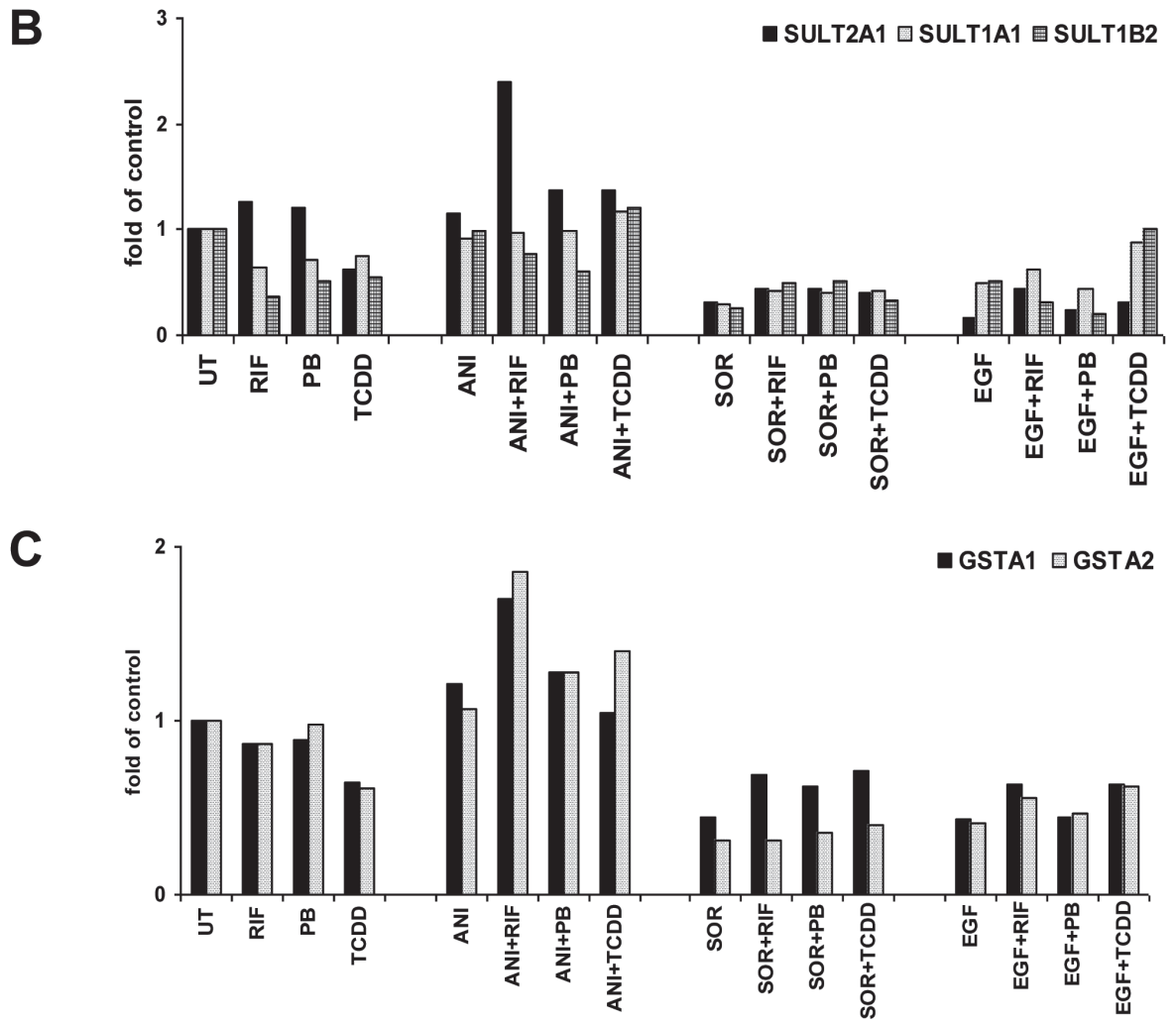

Figure 2. Effects of MAPKs activators on basal and inducible expression of UGT1A1, UGT2B17, SULT1A1, SULT2A1, SULT1B2, GSTA1 and GSTA2 mRNAs in human hepatocytes. Shown are RT-PCR analyses of UGT1A1, UGT2B17 (A), SULT1A1, SULT2A1, SULT1B2 (B), GSTA1 and GSTA2 (C) mRNAs from human hepatocytes culture FT278. The data are mean from duplicate measurements and are expressed as fold induction over DMSO-treated cells. The data were normalized to GAPDH mRNA levels. UT, untreated cells.

EGF, whereas ANI potentiated SULT2A1 induction by RIF (Fig. 2B). Expression of GSTA1 and GSTA2 was not affected by RIF and PB, while TCDD decreased the levels of both mRNAs. Combined treatments with SOR and EGF plus inducers displayed similar pattern as in the absence of inducers. In contrast, ANI significantly augmented the levels of GSTA1 and GSTA2 in the presence of inducers (Fig. 2C).
Finally, we analyzed the effects of MAPKs activators on the expression of the receptors involved in the transcriptional control of biotransformation enzymes. The levels of AhR, GR, CAR and PXR were drastically reduced by SOR and EGF, while ANI decreased only CAR mRNA (Fig. 3).

In the present paper we show that MAPKs are involved in transcriptional regulation of important phase I and 
- AhR IGR GPXR 四AR

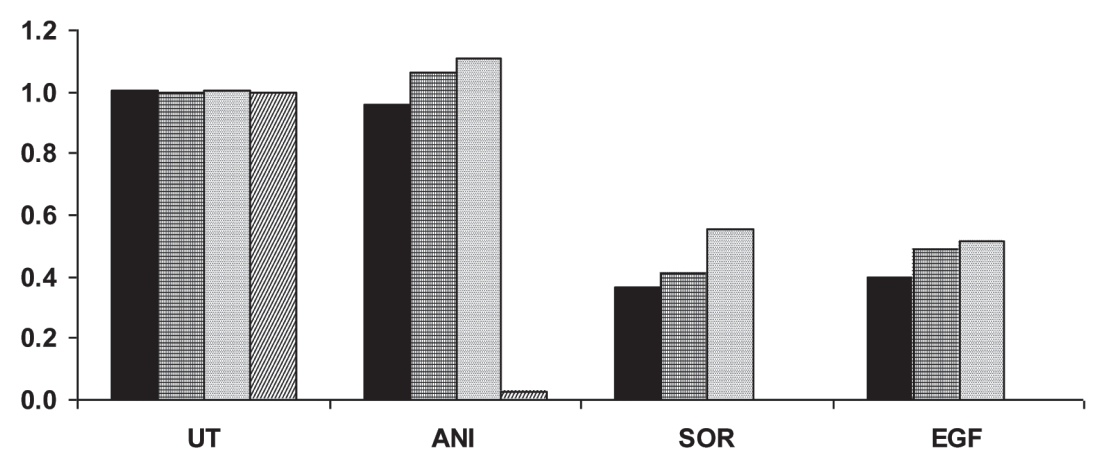

Figure 3. Effects of MAPKs activators on the expression of AhR, PXR, CAR and GR in human hepatocytes. Shown are RT-PCR analyses of AhR, PXR, CAR and GR mRNAs from human hepatocytes culture FT278. The data are mean from duplicate measurements and are expressed as fold induction over DMSO-treated cells. The data were normalized to GAPDH mRNA levels. UT, untreated cells.

phase II biotransformation enzymes in human hepatocytes. This is supported by the observation that model activators of MAPKs affected basal and inducible expression of drug-metabolizing enzymes. MAPKs activators displayed differential effects; SOR and EGF exerted in general inhibitory activity against basal and inducible expression of drug-metabolizing enzymes, whereas ANI had either no or slightly stimulatory effects. We may anticipate differential role of c-Jun-N-terminal kinase and extra cellular-activated protein kinase in constrast with $\mathrm{p} 38$, when considering specific activation of the either kinase by an appropriate activator. Howevere, we shown elsewhere that the effects of ANI and EGF on the activation of MAPKs in human hepatocytes are not specific in contrast to the majority of cell lines (Henklova et al. 2008a). In addition, the inhibitory effect of ANI on proteosynthesis should be taken in account, since it was described that the inhibitors of protein synthesis cause a superinduction of CYP1A1 gene (Joiakim et al. 2004). Collectively, we report here for the first time on the effects of MAPKs on transcriptional regulation of drug metabolizing enzymes in primary human hepatocytes.

Acknowledgement. Our laboratory is supported by the grants from the Czech Scientific Foundation GACR303/07/0128 and GACR305/08/P089.

\section{References}

Bogoyevitch M. A., Ketterman A. J., Sugden P. H. (1995): Cellular stresses differentially activate c-Jun $\mathrm{N}$-terminal protein kinases and extracellular signal-regulated protein kinases in cultured ventricular myocytes. J. Biol. Chem. 270, 29710-29717; doi:10.1074/jbc.270.50.29710
Donato M. T., Lahoz A., Castell J. V., Gomez-Lechon M. J. (2008): Cell lines: a tool for in vitro drug metabolism studies. Curr. Drug Metab. 9, 1-11; doi:10.2174/ 138920008783331086

Henklova P., Vrzal R., Papouskova B., Bednar P., Jancova P., Anzenbacherova E., Ulrichova J., Maurel P., Pavek P., Dvorak Z. (2008a): SB203580, a pharmacological inhibitor of p38 MAP kinase transduction pathway activates ERK and JNK MAP kinases in primary cultures of human hepatocytes. Eur. J. Pharmacol. 593, 16-23; doi:10.1016/ j.ejphar.2008.07.007

Henklova P., Vrzal R., Ulrichova J., Dvorak Z. (2008b): Role of mitogen-activated protein kinases in aryl hydrocarbon receptor signaling. Chem. Biol. Interact. 172, 93-104; doi:10.1016/j.cbi.2007.12.005

Joiakim A., Mathieu P. A., Elliott A. A., Reiners J. J. (2004): Superinduction of CYP1A1 in MCF10A cultures by cycloheximide, anisomycin, and puromycin: a process independent of effects on protein translation and unrelated to suppression of aryl hydrocarbon receptor proteolysis by the proteasome. Mol. Pharmacol. 66, 936-947

Kyriakis J. M., Avruch J. (1996): Sounding the alarm: protein kinase cascades activated by stress and inflammation. J. Biol. Chem. 271, 24313-24316; doi:10.1074/ jbc.271.40.24313

Oesch-Bartlomowicz B., Oesch F. (2008): Phosphorylation of xenobiotic-metabolizing cytochromes P450. Anal. Bioanal. Chem. 392, 1085-1092; doi:10.1007/s00216008-2315-2

Pascussi J. M., Gerbal-Chaloin S., Duret C., Daujat-Chavanieu M., Vilarem M. J., Maurel P. (2008): The tangle of nuclear receptors that controls xenobiotic metabolism and transport: crosstalk and consequences. Annu. Rev. Pharmacol. Toxicol. 48, 1-32; doi:10.1146/annurev. pharmtox.47.120505.105349

Pavek P., Dvorak Z. (2008): Xenobiotic-induced transcriptional regulation of xenobiotic metabolizing enzymes of the 
cytochrome P450 superfamily in human extrahepatic tissues. Curr. Drug Metab. 9, 129-143; doi:10.2174/ 138920008783571774

Roberts P. J., Der C. J. (2007): Targeting the Raf-MEK-ERK mitogenactivated protein kinase cascade for the treatment of cancer Oncogene 26, 3291-3310; doi:10.1038/sj.onc. 1210422

Vrzal R., Daujat-Chavanieu M., Pascussi J. M., Ulrichova J., Maurel P., Dvorak Z. (2008): Microtubules-interfering agents restrict aryl hydrocarbon receptor-mediated CYP1A2 induction in primary cultures of human hepatocytes via $\mathrm{c}$-jun-N-terminal kinase and glucocorticoid receptor. Eur. J. Pharmacol. 581, 244-254; doi:10.1016/ j.ejphar.2007.11.059

Received: October 23, 2008

Final version accepted: June 11, 2009 